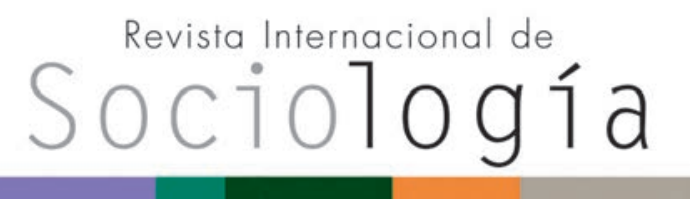

Revista Internacional de Sociología RIS

vol. 76 (3), e107, julio-septiembre, 2018, ISSN-L:0034-9712

https://doi.org/10.3989/ris.2018.76.3.17.12

\section{ABSTENCIÓN SEXUAL DURANTE LA CUARESMA EN ANDALUCÍA A LO LARGO DEL SIGLO XX Y SU IMPACTO EN LA ESTACIONALIDAD DE LOS NACIMIENTOS}

Josep LLEDó

Universitat de València

jonabe@alumni.uv.es

ORCID iD: https://orcid.org/0000-0002-7475-8549

Carles X. Simó-Noguera

Universitat de València

carles.simo@uv.es

ORCID iD: https://orcid.org/0000-0002-3569-1804

Jose M. Pavía

Universitat de València

pavia@uv.es

ORCID iD: https://orcid.org/0000-0002-0129-726X

Cómo citar este artículo / Citation: Lledó, J., C. X. Simó-Noguera y J. M. Pavía. 2018. "Abstención sexual durante la Cuaresma en Andalucía a lo largo del siglo $\mathrm{XX}$ y su impacto en la estacionalidad de los nacimientos". Revista Internacional de Sociología 76(3): e107. https://doi.org/10.3989/ris.2018.76.3.17.12

\footnotetext{
Resumen

Durante siglos ha permanecido vigente en las poblaciones católicas un precepto religioso que prohibía las relaciones sexuales durante la Cuaresma. Esta interdicción repercutía en una disminución de concepciones y en un repunte tras dicho periodo, ambos difíciles de detectar porque, en poblaciones que no ejercían un control efectivo de la fecundidad, también se registraba un pico de concepciones durante la primavera. En la actualidad este precepto ha desaparecido como consecuencia de un proceso de erosión que no conocemos suficientemente. Con los datos anonimizados de todas las personas nacidas en Andalucía y supervivientes a 1 de enero de 2003 ( $n=8.397 .206)$, este trabajo pretende determinar la importancia de esta interdicción y su desaparición a lo largo del siglo XX, coincidiendo con el descenso de la fecundidad y la desestacionalización de los nacimientos. Finalmente, también se analiza hasta qué punto esta transición hacia la modernidad es resultado de un proceso de difusión que ha ido desde municipalidades de gran tamaño poblacional a municipalidades pequeñas.
}

\section{Palabras Clave}

Concepción; Difusión; Erosión; Fecundidad; Preceptos religiosos.

\section{SEXUAL ABSTENTION DURING LENT IN ANDALUSIA ALONG THE 20TH CENTURY AND ITS IMPACT ON THE SEASONALITY OF BIRTHS}

Copyright: (C) 2018 CSIC. Este es un artículo de acceso abierto distribuido bajo los términos de la licencia de uso y distribución Creative Commons Reconocimiento 4.0 Internacional (CC BY 4.0).

Recibido: 23/01/2017. Aceptado: 07/06/2017

\section{Abstract}

A religious precept that forbids sexual intercourse during Lent has remained in effect for centuries in Catholic populations. This interdiction produces a decrease of conceptions and a rebound after that period, which are difficult to detect because in populations that did not exercise effective control of the fecundity a peak of conceptions is observed during the spring. At present, this precept has disappeared as a result of a process of erosion that we do not know enough. With the anonymized data of all the people surviving at the beginning of 2003 and born in Andalusia $(n=8,397,206)$, this paper aims to determine the importance of this interdiction and its vanishing process throughout the twentieth century. This process takes part with the decrease of fertility and the erosion of seasonality of births. Finally, it is also analyzed to what extent this transition to modernity is the result of a process of diffusion that has gone from municipalities with a big population size to small municipalities.

\section{KeYWORDS}

Conception; Diffusion; Erosion; Fertility; Religious precepts. 


\section{INTRODUCCIÓN}

Este trabajo estudia la importancia que ha podido tener el precepto religioso que prohibía las relaciones sexuales durante la Cuaresma en el siglo $X X$ mediante el estudio de la distribución, a lo largo del tiempo, de las concepciones de las personas nacidas en Andalucía que se encontraban con vida y residían en España a fecha 1 de enero de 2003. A partir de las fechas de nacimiento se estiman las fechas de concepción y se estudia el cambio de intensidad, medido en ratios, que experimentan las concepciones antes, durante y después del periodo de Cuaresma.

Los cambios de intensidad se analizan junto con el patrón dominante de la estacionalidad de los nacimientos y el cambio que la estacionalidad ha experimentado a lo largo del siglo XX, desde un modelo que la literatura ha caracterizado como regulado por factores ambientales, a otro condicionado por factores socioculturales (Quesada 2006). La finalidad es observar cómo se ha ido erosionando el respeto a este precepto religioso en un siglo de profundas transformaciones en las pautas reproductivas y de descenso de la fecundidad a umbrales inferiores al nivel de reemplazamiento generacional. Mediante el análisis con técnicas de series temporales se demuestra la existencia del efecto Cuaresma durante gran parte del siglo XX, y se prueba su posterior desaparición.

Esta investigación se integra en el debate sobre la influencia que ejerció la religión católica en materia de sexualidad y reproducción, al ocuparse de la incidencia que puede haber tenido en las concepciones la interdicción religiosa de mantener relaciones sexuales durante la Cuaresma, y del proceso que ha llevado a la total desaparición del cumplimiento de este precepto religioso. Para ello, se emplean las técnicas estadístico-econométricas más avanzadas de tratamiento de series temporales que complementan y, en cierta medida, suponen un avance respecto a los estudios clásicos sobre la estacionalidad de los nacimientos.

El origen histórico de esta interdicción data de los siglos $\mathrm{VI}$ a XI, periodo durante el cual los penitentes conformaron la doctrina oficial de la iglesia católica en torno a las relaciones sexuales y la reproducción, y establecieron diferentes periodos de interdicción de las relaciones sexuales. Uno de los periodos más destacados en la prohibición en las relaciones sexuales es la Cuaresma, periodo que comprende los 47 días anteriores a la Pascua de Resurrección (Bazán 2008; Cabanes 2003).

El alcance de esta interdicción religiosa es de especial interés para profundizar en el estudio de la transición de la fecundidad en Andalucía. Aunque gran parte de la reducción de la natalidad que se produjo durante la transición demográfica tuvo lugar antes de que los métodos anticonceptivos se convirtieran en herramientas eficaces de control de la fecundidad y devinieran accesibles a la mayoría de la población, nada o poco sabemos sobre (i) cuál ha sido la importancia y el alcance efectivo que tuvo esta interdicción religiosa entre las parejas fértiles; (ii) hasta cuándo ha estado presente este fenómeno y (iii) cuándo y cómo se ha ido erosionando.

En primer lugar, se repasan las aportaciones teóricas que han permitido conocer las pautas reproductivas del pasado y sobre las que se sustentan las preguntas de investigación. Con este propósito, se integran dos temáticas muy relacionadas entre sí y profusas en trabajos empíricos. El debate sobre la capacidad de determinar la descendencia, así como el debate sobre la estacionalidad de los nacimientos en poblaciones sin control efectivo de la fecundidad, son necesarios para enmarcar el proceso de transición de la fecundidad subyacente en nuestro periodo de estudio. A continuación se introducen las hipótesis de investigación, los datos utilizados y las técnicas de análisis. Posteriormente se presentan los resultados y finalmente se ofrecen las conclusiones.

\section{PaUtAs REPRODUCTIVAS PREVIAS AL CONTROL DE LA FECUNDIDAD $Y$ ESTACIONALIDAD DE NACIMIENTOS}

En poblaciones históricas, el análisis de los intervalos genésicos, el estudio de la fecundidad por duración de la unión y por edad de la madre y el análisis de la fecundabilidad ${ }^{1}$, mediante la medición del tiempo que separa el matrimonio del nacimiento del primer hijo, han permitido conocer la intensidad de la fecundidad y el espaciamiento de los nacimientos a lo largo de la vida fecunda de los matrimonios. Los estudios han concluido la existencia de una incapacidad generalizada de intervención efectiva de las parejas en la anticoncepción y, principalmente, una falta de control sobre el número de hijos habidos (Iriso y Reher 1987; Henry 1961).

Diferentes áreas de las ciencias sociales han estudiado hasta qué punto las personas deciden sobre su proyecto reproductivo o son capaces de intervenir en él (Liefbroer et al. 2015; Lesthaeghe 1980; Reher 1988). Esto también es especialmente relevante en poblaciones como las históricas. El demógrafo Louis Henry propuso el concepto de fecundidad natural en ausencia de un control deliberado de los nacimientos (Henry 1961). Este concepto asumía la intervención de factores sociales que podían conducir a una reducción de la fecundidad, pero que no podían ser considerados una forma deliberada de control. La fecundidad deja de ser "natural" cuando la intervención de la pareja está determinada por el número de hijos deseados (Henry 1961). Para Coale (1973), una de las condiciones para que se dé la transición de la fecundidad es que las decisiones sobre la reproducción han de formar parte de un cálculo de elección consciente. De hecho, en el proceso de moderniza- 
ción de las pautas reproductivas, las personas buscaron un mayor espacio para la elección individual en respuesta a las nuevas condiciones, al tiempo que se enfrentaban a un código moral y unas formas tradicionales de control (Lesthaeghe 1980: 434-535).

Hoy en día sabemos que gran parte de la reducción de la fecundidad, en Europa y en España, tuvo lugar antes de que los métodos anticonceptivos y los abortos inducidos con apoyo médico fueran realmente accesibles para la población (Ruiz et al. 2005). Gracias al método de reconstrucción de familias se ha podido analizar el descenso de la fecundidad que tuvo lugar en nuestro territorio antes de la revolución anticonceptiva. Destacan, entre otros, los estudios de Sánchez (2006) sobre tres localidades de la provincia de Navarra; Torrents (1995) y Masdéu (2015) sobre dos municipios catalanes; o Ramírez (1998) sobre dos municipios del interior andaluz. También se conoce que, históricamente, las parejas que limitaban el tamaño de la descendencia lo hacían, en su mayoría, mediante el coitus interruptus o la abstinencia (Knodel y Van de Walle 1979), y que existieron mecanismos de intervención en la reproducción (Bentley et al. 1993) de naturaleza muy diversa que pueden o no revelar un carácter deliberado (Lesthaeghe 1980).

Junto con los estudios biométricos de la fecundidad, existen numerosos trabajos que demuestran la existencia de pautas culturales y medioambientales que también interferían en la reproducción. El análisis de la estacionalidad de los nacimientos ha aportado evidencias sobre la existencia de patrones de comportamiento anual no uniformes, que muestran la presencia de mecanismos de intervención o de factores externos.

En un esfuerzo por sintetizar las diferentes orientaciones existentes, Ellison, Valeggia y Sherry (2005) hablan de tres grupos de factores en la estacionalidad de las concepciones: (i) los factores sociales que influyen en la frecuencia de las relaciones sexuales, (ii) los factores climatológicos que afectan a la fertilidad humana, y (iii), finalmente, los factores energéticos que influyen en la fertilidad femenina. Entre los dos primeros grupos de factores, y centrados en poblaciones del pasado, la literatura señala: 1) la incidencia del fotoperiodo en la fertilidad de los mamíferos, que determina una mayor probabilidad de concebir en primavera, 2) la existencia de un calendario agrícola con estaciones en las que los nacimientos o las concepciones eran más o menos deseadas u oportunas, y 3) el calendario religioso, que exhortaba a la abstención de mantener relaciones sexuales durante la Cuaresma y el Adviento.

El fotoperiodo y la temperatura son señalados como los factores climatológicos que influyen en la fertilidad de los mamíferos (capacidad biológica de procrear) (Ellison et al. 2005). El hecho de que la producción de espermatozoides entre mamíferos sea sensible a la temperatura ambiente y de que se optimice a temperaturas por debajo de la temperatura corporal media, ha sido objeto de interés de muchas investigaciones. Algunas evidencias apoyan esta relación. Por ejemplo, algunos países con clima caluroso presentan un mayor número de concepciones en invierno, mientras que en las zonas templadas el mayor número de concepciones se localiza en verano (Lam y Miron 1994; Rusell, Douglas y Allan 1993). También existen estudios que señalan que las altas temperaturas reducen las concepciones en una amplia variedad de poblaciones (Lam y Miron 1991).

El fotoperiodo también afecta a la fertilidad humana (Wehr 1991). La duración media de las horas de luz influye en la estacionalidad de las concepciones, puesto que la secreción de melatonina por parte de la glándula pineal es fotosensible y, en los humanos, ocurre principalmente en la noche (Wehr et al. 1993). Esta hormona es importante en el mantenimiento de los ciclos circadianos y los patrones estacionales de reproducción de muchos mamíferos. Varias investigaciones han relacionado los picos de la estacionalidad de los nacimientos con el fotoperiodo. En un estudio realizado en Andalucía se ha observado una mayor probabilidad de concebir en primavera, relacionada con el mecanismo hormonal modulado por el fotoperiodo (Quesada 2006). De la misma manera, un estudio de la estacionalidad de los nacimientos en España entre 1941 y 1960 ha mostrado un gran pico en las concepciones de primavera, asociado al fotoperiodo; y un menor pico en otoño (CanchoCandela, Andrés-de Llano y Ardura-Fernández 2007). Obviamente, esa podría no ser la única explicación, pues un mayor número de concepciones en primavera también sería compatible, en el caso de Andalucía, con la hipótesis de calendario agrícola.

De hecho, en la península ibérica contamos con estudios que relacionan la estacionalidad de los nacimientos en poblaciones pre-modernas con el calendario agrícola, y sugieren que este calendario permitía concentrar los nacimientos en el punto álgido del invierno (Gual 1988: 29-30). Como advierte Sánchez Barricarte (2006: 450), el invierno era el mejor momento para cuidar a las mujeres en el parto y cuidar a los recién nacidos; además, al ser menor el número de nacimientos en verano y otoño, las mujeres se encontraban más libres para ayudar a sus maridos en el campo. Según Gual, con este calendario convergían dos ventajas: había poca actividad en el campo y había una menor incidencia de la mortalidad infantil (Gual 1988: 187). Algunos demógrafos también han analizado la relación entre el mes de nacimiento y la supervivencia de los recién nacidos (Breschi y LiviBacci 1994). Los datos recogidos hasta ahora para la Cataluña rural también coinciden con la hipótesis del calendario agrícola hasta el siglo XIX: un máximo de concepciones entre abril y junio y unos mínimos entre agosto y noviembre (Masdéu 2015: 414). 
Otros autores señalan que el tamaño de la región estudiada condiciona de forma notable los factores sobre la estacionalidad de los nacimientos (Fuster 1989). Sin embargo, estos patrones culturales de las concepciones interaccionan fuertemente con limitaciones ecológicas en poblaciones con regímenes de subsistencia (Condon y Scaglion 1982). Además, la relación entre factores culturales y factores climatológicos y/o de ingesta energética es muy estrecha (Ellison, Valeggia y Sherry 2005) y difícil de disociar.

Finalmente, cabe apuntar la influencia del calendario religioso. En el pasado, la iglesia católica ejerció un papel importante en la reproducción. Prohibió la sexualidad fuera del matrimonio, condenó las relaciones sexuales cuya finalidad no fuera la concepción y estableció periodos de interdicción de las relaciones sexuales, como el Adviento y la Cuaresma (Bazán 2008; Cabanes 2003). Diferentes estudios han probado la existencia de fuertes normas religiosas en culturas católicas y calvinistas (por ejemplo, Lesthaeghe y Wilson 1986). En nuestro país, la influencia de la religión católica en las pautas reproductivas ha sido notable hasta muy recientemente. En su estudio sobre la fecundidad en España en el periodo 1887-1920, Iriso y Reher (1987) elaboran un indicador con la intención de relacionar el proceso de secularización con la transición a la fecundidad y, aunque no encuentran el efecto esperado, sugieren que la propagación de las actitudes secularizadas fue clave en la reducción consciente de la fecundidad. Las doctrinas de la iglesia católica constituyeron un apoyo de gran importancia para la existencia del sistema demográfico en las sociedades preindustriales, caracterizado por una alta fecundidad (Iriso y Reher 1987).

Un estudio sobre la evolución de la práctica religiosa en los últimos tres decenios del siglo $\mathrm{XX}$ muestra una disminución del nivel de creencias y prácticas religiosas y, sobre todo, la existencia de una amplia desconexión entre creencias, prácticas y aceptación de la normativa eclesial (DíazSalazar 1993). Según datos aportados por DíazSalazar, son especialmente los jóvenes que tenían entre 15 y 25 años en 1975 los que introducen una reducción de cerca del $50 \%$ en la práctica religiosa semanal respecto a 1960. Más recientemente, en un estudio sobre el proceso de secularización, Requena (2005) analiza la disminución de los niveles de la práctica religiosa y prueba que este proceso fue favorecido por factores estructurales como la edad y el sexo, alcanzando a todas las categorías sociales relevantes de la sociedad española desde, al menos, 1975. Por otro lado, algunas evidencias parecen sugerir la permanencia de una cierta influencia religiosa en las pautas reproductivas. De hecho, se ha podido probar que en 1999 los matrimonios que se declaraban cató- licos practicantes tuvieron una fecundidad mayor que los que se declaraban no practicantes (Adserà 2006). Como señala Lesthaeghe (1980: 534-535), en la transición de la fecundidad es clave entender cómo se modifica y diversifica el sistema cultural en un contexto de mayor necesidad de una toma de decisiones individualizada; y en este sistema el papel de la religión es clave.

En la actualidad las parejas españolas deciden en gran manera sobre su proyecto reproductivo y estas decisiones conciernen tanto al número de hijos que se quieren tener, como cuándo y cómo concebirlos (Cordero 2009). Como consecuencia de ello, actualmente la distribución anual de los nacimientos está muy influenciada por el efecto de la planificación de la concepción. En esta misma línea insiste Haandrikman (2004) al señalar la profunda transformación que, en Holanda, ha experimentado la estacionalidad de los nacimientos y la importancia que ha tenido la planificación consciente de los embarazos. Estudios recientes prueban que la organización hospitalaria ha modificado la distribución semanal de nacimientos (Lledó, Pavía y Morillas 2017). Son, pues, varios los autores que apuntan a un cambio definitivo en los factores relacionados con la estacionalidad de los nacimientos. Por ejemplo, Bobak y Gjonca (2001) analizan los nacidos vivos en el periodo 1989-1991 en la República Checa y muestran que los partos de las madres entre 25 y 34 años, casadas y con estudios superiores, presentan una mayor estacionalidad.

Así pues, los factores sociodemográficos que influyen actualmente en la estacionalidad de los nacimientos poco tienen que ver con los factores del pasado. Quesada (2006), en su estudio sobre Andalucía, encuentra evidencias del cambio que tuvo lugar durante el siglo $\mathrm{XX}$, cuando se pasó de un modelo donde la estacionalidad de los nacimientos estaba regulada por el medio ambiente a otro determinado por condicionantes de tipo sociocultural. Sin embargo, no sólo actuaba el medioambiente en la estacionalidad pre-moderna de las concepciones. Otros factores, como el fotoperiodo y el calendario agrícola, se conjugaban con la cronología de los preceptos religiosos que afectaba tanto a los matrimonios como a las concepciones. Tal como apuntan Reher y Sanz (2006: 107), en la literatura demográfica se ha tendido a considerar que la estacionalidad de los matrimonios y de los nacimientos es producto de fuerzas religiosas y/o socio-económicas, mientras que la estacionalidad de las defunciones se ha relacionado con factores relacionados con el clima. La Figura 1 muestra que los patrones estacionales de las concepciones anteriores a 1960 y posteriores a 1980 en Andalucía son claramente distintos y que, además, las fluctuaciones estacionales se han reducido significativamente (más de la mitad). 
Figura 1.

Componente estacional de la distribución mensual de las concepciones en Andalucía. Panel derecho: 1938-1960. Panel izquierdo: 1980-2002.

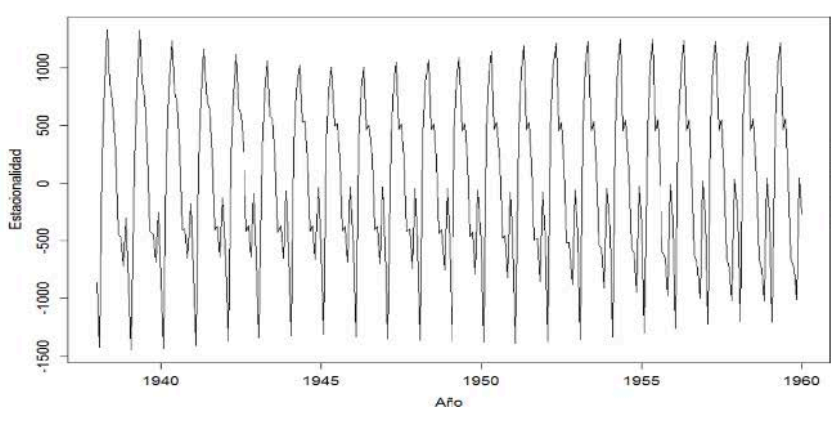

\section{Preguntas de inVestigación, datos Y TÉCNICAS}

En el estudio del papel ejercido por la religión en la estacionalidad de los nacimientos nos proponemos dar respuesta a las siguientes preguntas de investigación: (i) ¿Hasta qué punto es importante la penuria de concepciones durante la Cuaresma?; (ii) ¿Qué datación sigue la erosión del cumplimiento del precepto religioso de mantener relaciones sexuales durante la Cuaresma en Andalucía?, y (iii) Esta erosión, ¿siguió un proceso de difusión desde ámbitos municipales de gran tamaño a ámbitos municipales pequeños, tal como ha sucedido con otros procesos en la transición a la modernidad?

Para responder a tales preguntas se introducen las siguientes argumentaciones o hipótesis de investigación: (1) A lo largo de gran parte del siglo XX, los nacimientos en Andalucía se distribuyen según un patrón de estacionalidad con picos y valles propios de las sociedades pre-modernas, visibles en los trabajos desarrollados hasta el momento. (2) Durante ese mismo periodo, la influencia de la religión católica en las pautas reproductivas de la población andaluza fue importante hasta el punto que es espera encontrar un menor número de concepciones durante el periodo de Cuaresma. Profundizando en el análisis, en los primeros setenta años del siglo $\mathrm{XX}$, (3) se puede esperar un fuerte repunte, tras el periodo de 47 días de interdicción religiosa, en el número de concepciones, al tratarse de una sociedad prácticamente sin acceso a técnicas anticonceptivas ni a la planificación efectiva de los nacimientos. En lo que respecta a los últimos años del pasado siglo se produce un cambio sustancial y (4) la estacionalidad de los nacimientos obedece ya al control efectivo de la fecundidad, la planificación de las concepciones y la secularización de la sociedad. Finalmente, acorde con los estudios sobre la difusión de la modernización, (5) el cambio se dio antes en ámbitos municipales de gran tamaño que en municipios de pequeño tamaño poblacional.

La información utilizada en este estudio son las fechas de nacimiento (día, mes y año) de la pobla-

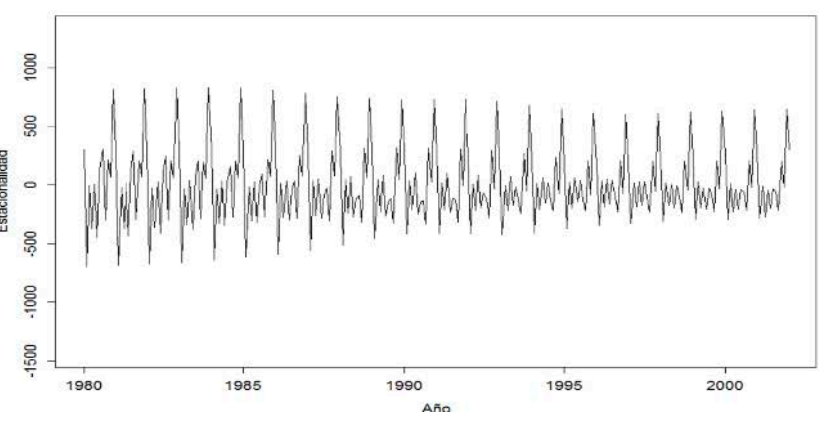

ción andaluza en los últimos 100 años y con vida a 1 de enero de 2003, datos anonimizados contenidos en el Padrón Municipal de Habitantes de España $(n=8.397 .206)$. Los datos utilizados fueron adquiridos previo pago al Instituto Nacional de Estadística (INE). La información utilizada puede considerarse adecuada y fiable, ya que al no existir relación alguna entre las personas nacidas y fallecidas entre el periodo de 1901 a 2002 y su día de nacimiento no puede darse ningún efecto de selección. Una vez conocido el momento del nacimiento, estimamos el momento de la concepción.

Dada la gran dificultad que conlleva el conocimiento del momento exacto de la concepción de un individuo, presumimos que dicho momento se sitúa 280 días (9 meses y 10 días) antes del momento del nacimiento. En nuestro estudio asumimos que los nacimientos prematuros y los partos múltiples (prematuridad) tienen una influencia estadística limitada y que ésta, en todo caso, no obedece a un comportamiento estacional diferencial de concepciones. Además, contamos con un número de casos suficientemente grande como para asumir que estos nacimientos no perjudican nuestros resultados. Aun así, algunos análisis son realizados, concretamente en el cálculo de ratios, eliminando el efecto nacimientos prematuros.

Para observar la difusión de la erosión de este precepto religioso según el tamaño de hábitat, hemos empleado una distinción operativa entre municipios de gran tamaño y municipios pequeños, puesto que, en la actualidad, no existe un consenso sobre la delimitación de los contornos urbanos y rurales (OECD 2012). Goerlich y Cantarino (2015) siguen una tipología que relaciona la densidad de población con el tamaño poblacional para el caso español. Otros estudios parten del espacio urbano y la distancia en tiempo de conducción para catalogar el espacio rural (Dijkstra y Poelman 2008). Nuestra propuesta consiste en maximizar el tamaño de hábitat como criterio distintivo y diferenciar entre hábitat de pequeño (poblaciones con menos de 10.000 habitantes) y de gran tamaño (poblaciones con más de 50.000)². Con estos límites pretendemos minimizar el efecto de 
las modificaciones que hayan podido sufrir los municipios desde el momento del nacimiento, ya que la fuente de datos utilizada consigna el tamaño del municipio en el momento de la petición de los datos.

Puesto que el emplazamiento de la Cuaresma no sigue el calendario solar, se han buscado los inicios y los finales del periodo de Cuaresma para todos los años analizados. Así, definimos el momento $t$ como el Domingo de Resurrección (final del periodo de Cuaresma) y $t-47$ como el inicio de dicho periodo (00:00 horas del Miércoles de Ceniza). Las técnicas estadísticas empleadas para dar respuesta a las preguntas de investigación se diferencian en dos grupos. En primer lugar, estudiamos la serie histórica mensual del número de concepciones mediante técnicas propias de series temporales (Box y Jenkins 1976; Uriel y Peiró 2000). En un segundo análisis se comparan periodos homogéneos de tiempos anteriores, simultáneos, o posteriores al periodo de Cuaresma y se calculan ratios estadísticos, cuyo objetivo principal es identificar patrones en las concepciones en periodo de Cuaresma durante todo el siglo XX.

Las concepciones (los nacimientos) muestran un fuerte componente estacional que, por una parte, domina las fluctuaciones de corto plazo de la serie (ver Figura 1 y Figura 2, derecha) y, por otra, ha evolucionado a lo largo del tiempo (ver Figura 1). En estas condiciones, la estimación de los índices de variación estacional mediante una metodología clásica, basada en filtros fijos (como, por ejemplo, el cociente entre el número medio de nacimientos en cada mes y el número medio estandarizado de nacimientos anuales ${ }^{3}$ ), tiene el inconveniente de que no permite detectar la presencia de otros efectos de menor intensidad y con un emplazamiento cambiante año a año (como es el caso de la Cuaresma).

Por ello, para el tratamiento mediante series temporales ${ }^{4}$, hemos empleado las técnicas estadísticoeconométricas (US Census Bureau 2016) más avanzadas, que se benefician de los más de 40 años de progresos metodológicos desarrollados dentro del área del análisis económico coyuntural en el que, para extraer el perfil de evolución subyacente de las series, el analista debe resolver situaciones con componentes estacionales no estacionarios y con efectos de calendario no sistemáticos (como, por ejemplo, el número de sábados de un mes o el porcentaje de días de semana santa de cada mes) que, aun siendo de una magnitud significativamente menor a las fluctuaciones estacionales, pueden influir en la evolución de la serie.

Las variaciones estacionales son fluctuaciones que ocurren en cada subperiodo del año siguiendo un patrón regular (por ejemplo, San Valentín siempre es el 14 de febrero o las bodas siempre tienden a concentrarse en los mismos meses del año) y quedan recogidas en el componente estacional. Los efectos de calendario, por su parte, se definen como el impacto que produce en la serie temporal la estructura inconstante que presentan las variables que pueden impactar en la evolución temporal de la serie (tanto en longitud como en composición) en los distintos meses de los diferentes años. Es precisamente esta estructura inconstante lo que permite disociar los efectos de calendario de las variaciones estacionales. Por ejemplo, el ciclo semanal se da como consecuencia de que no todos los meses tienen el mismo número de fines de semana (lo que podría afectar al número de concepciones); mientras la Cuaresma, al seguir un patrón basado en el calendario lunar, fluctúa de un año a otro en ubicación respecto a los meses, que responden al calendario solar.

Por lo que, de existir un efecto Cuaresma en las concepciones, éste debería depender del número de días de Cuaresma que tiene cada mes. Es decir, una vez descontado el efecto estacional y el posible efecto del ciclo semanal, debería esperarse un menor número de concepciones en los meses con más días de Cuaresma, si se cumple con el precepto religioso que prohibía las relaciones sexuales. En otras palabras, el coeficiente asociado al regresor temporal de la Cuaresma debería ser estadísticamente significativo y con el signo apropiado. Así, utilizamos modelos regARIMA (modelos autorregresivos y de medias móviles con regresores) para estimar simultáneamente, por máxima verosimilitud, el conjunto de parámetros del modelo (US Census Bureau 2016) y estudiar, junto con el componente estacional, el posible impacto de efectos de calendario, como la Cuaresma y el ciclo semanal.

Por otro lado, desde una perspectiva más descriptiva, se han computado una batería de ratios. El ratio 1 , denotado como $r_{1}$, compara las concepciones registradas en el periodo de Cuaresma con el periodo de la misma amplitud inmediatamente anterior. El ratio 2 , denotado como $r_{2}$, compara las concepciones registradas en el periodo de Cuaresma con el periodo equivalente inmediatamente posterior. De acuerdo con una hipótesis de erosión del efecto de la Cuaresma y de efectos estacionales ambientales, se espera una tendencia creciente para el ratio $1 \mathrm{y}$ una tendencia decreciente para el ratio 2 , con una progresiva convergencia a la unidad en los años finales del siglo XX. Con el objeto de facilitar la comprensión de todos los ratios utilizados para comparar cada uno de los momentos temporales, la Tabla 1 presenta tanto la formulación como los gráficos correspondientes. En la Tabla 1, conc $C_{T}$ denota las concepciones correspondientes al día $T$-ésimo.

La prohibición de tener relaciones sexuales durante el periodo de Cuaresma puede evidenciar un repunte, o efecto rebote, en el número de concepciones una vez terminado dicho periodo. El momento de la concepción es, sin embargo, estimado, por lo que la ubicación de las concepciones dentro o fuera de dicho periodo está sujeta a incertidumbre, debido a la anticipación por nacimiento prematuro. Según 
Tabla 1.

Detalle de los ratios calculados

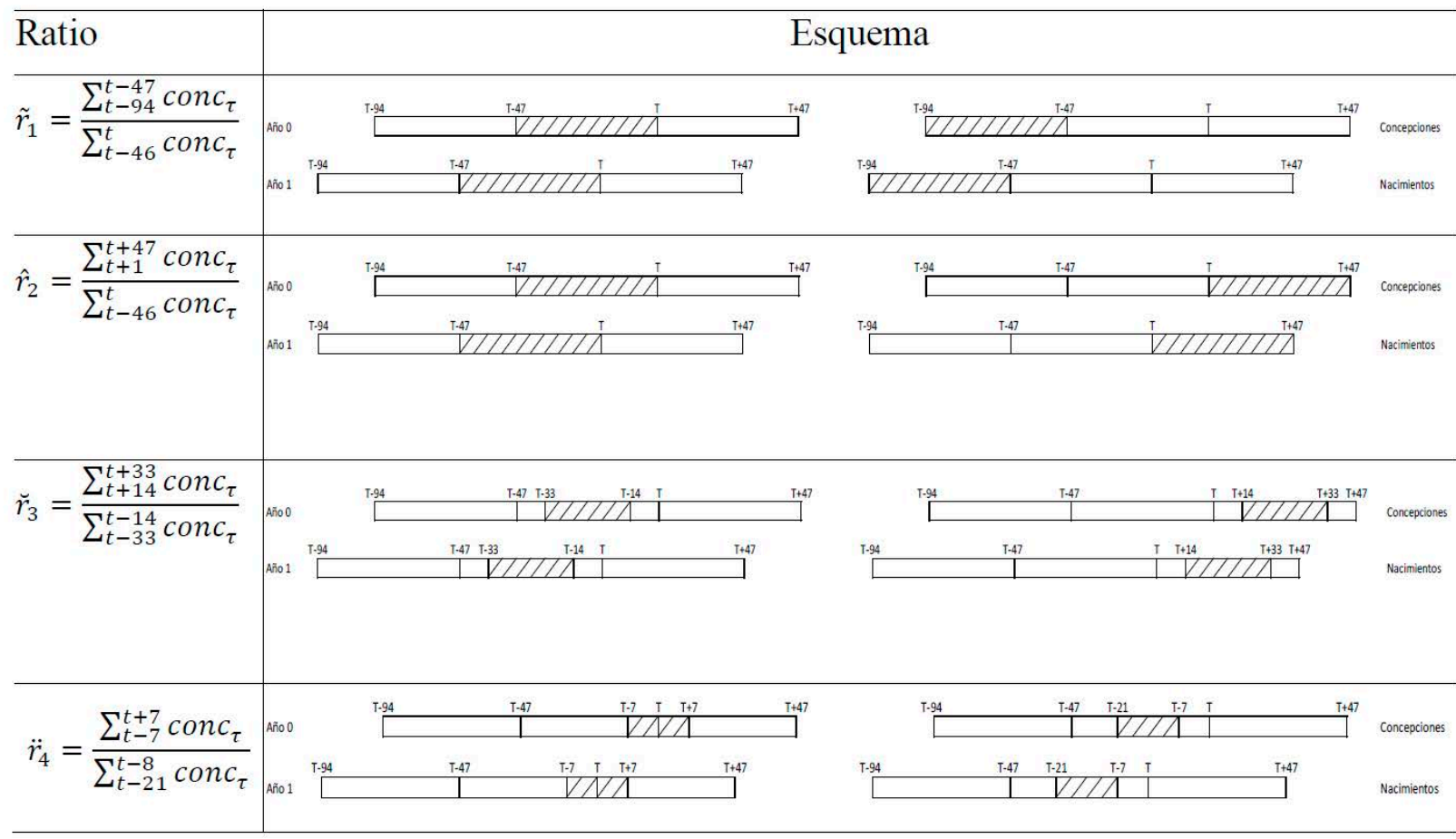

la Organización Mundial de la Salud, actualmente la tasa de nacimientos prematuros oscila entre el $5 \%$ y el $18 \%$ de los recién nacidos (OMS 2012). Para complementar los resultados de los dos ratios anteriores se han creado los ratios 3 y 4 . El ratio 3 , denotado como $r_{3}$, compara las concepciones del núcleo central (de 21 días) de Cuaresma con un periodo de igual longitud posterior a la finalización del periodo de Cuaresma y diferido en siete días, para minimizar el efecto de los partos prematuros. Un valor superior a la unidad en este ratio significaría un mayor número de concepciones una vez finalizado el periodo de abstinencia. Dado que se espera observar una abstinencia durante el periodo de Cuaresma, el ratio 3 debería presentar valores superiores al ratio 2 al tener en cuenta el momento central de dicho periodo. Por otro lado, el ratio 4 , denotado como $r_{4}$, se ha creado con una doble finalidad. Por un lado, elimina el efecto nacimientos prematuros, al comparar las concepciones tenidas en cuenta durante el periodo entre 7 días antes y después de la Cuaresma y el periodo entre 21 y 7 días antes de finalizar el periodo de Cuaresma. Por otro lado, debilita el posible efecto estacional del fotoperiodo presente en otros ratios, dado que compara dos momentos temporales totalmente contiguos y relativamente cortos: sólo 14 días.

A fin de hacer más compresibles los resultados y las tendencias, cada ilustración de ratios incorpora un suavizado, con el objetivo de mejorar la observación de la tendencia de la serie. Este suavizado se crea ajustando los datos mediante técnicas de regresión local loess (Cleveland, Grosse y Shyu 1992).
El software libre estadístico $R$, versión 3.3.0 ( $R$ Core Team 2016) ha sido utilizado, mediante la programación de scripts ad-hoc, para tratar todos los datos y aplicar las técnicas estadísticas. Para la parte de los ratios se ha utilizado la librería ggplot2 (Wickham 2009), mientras que para la parte de las series temporales se ha utilizado la librería seasonal (Christoph 2016) que utiliza un interfaz con $X$-13ARIMA-SEATS (US Census Bureau 2016).

\section{Resultados}

La primera pregunta de investigación se refería a que, en gran parte del siglo XX en Andalucía, los nacimientos (las concepciones) se distribuían según un patrón de estacionalidad con picos y valles propios de las sociedades pre-modernas (Wrigley y Schofield, 1981; Knodel, 1988). En este patrón las concepciones son más altas de abril a junio y registran su nivel más bajo de agosto a noviembre. Este mismo resultado lo encontramos en nuestros datos a principios del siglo XX. Pero observamos, además, un profundo cambio: la distribución de "nacimientos (concepciones)" en Andalucía pasa de tener un patrón estacional claro durante la primera mitad del siglo XX (ver Figura 2, izquierda), a un patrón con una distribución de "nacimientos (concepciones)" más constantes a lo largo de los distintos meses del año (muy probablemente motivado por factores socioculturales). Como se observa en los paneles derechos de las Figuras 1 y 2 , las fluctuaciones se reducen sensiblemente, con lo que la 
estacionalidad tiende a la desaparición durante las últimas décadas del siglo $\mathrm{XX}$.

El principal objetivo de esta investigación es, sin embargo, ofrecer evidencia sobre la importancia que ha podido tener, en la configuración de esta estacionalidad tradicional, el cumplimiento del precepto religioso que prohibía mantener relaciones sexuales durante el periodo de Cuaresma. Por ello, creemos que es de gran interés en el campo de las ciencias sociales: (i) determinar el impacto de los valores religiosos en las pautas reproductivas, mediante el análisis del efecto que haya podido tener este precepto religioso en la estacionalidad; (ii) datar el momento en que se produce el cambio, y (iii) estudiar cómo se ha dado el proceso de difusión hacia la modernidad desde ámbitos municipales de gran tamaño poblacional hasta ámbitos municipales de pequeño tamaño.

\section{¿Existe un efecto Cuaresma?}

El cálculo del coeficiente clásico de estacionalidad para el periodo de estudio ilustra la profunda estacionalización presente históricamente en las concepciones en Andalucía y su progresivo proceso de desestacionalización (Figura 2, izquierda). El tamaño de las variaciones estacionales es capaz de enmascarar cualquier fluctuación debida al efecto Cuaresma. Por lo que, a fin de estudiar su presencia, utilizando técnicas de regresión de series temporales, hemos analizado la serie temporal mensual del número de concepciones (Figura 2, derecha), incluyendo como regresor el porcentaje de número de días de Cuaresma que corresponde a cada mes.

Debido a que la Cuaresma comprende un periodo muy largo (47 días), hemos estudiado su efecto considerando que la interdicción religiosa de mantener relaciones sexuales durante la misma no necesariamente incide de forma homogénea a lo largo de todo el periodo. Como es habitual cuando se estudia el efecto Pascua en una serie temporal (US Census Bureau 2016: 35-36), hemos estudiado para todos los números de días, desde 1 hasta 47 antes del Domingo de Resurrección, cuál es el efecto de incorporar el regresor. Asumiendo que el efecto Cuaresma se intensifica a medida que nos acercamos a la Semana Santa, se espera que el efecto sea estadísticamente más significativo cuanto más próximos esté el Domingo de Resurrección. Asimismo, debido a que la serie presenta dos patrones estacionales claramente distintos (ver Figura 1), hemos ajustado las regresiones para diferentes periodos: hasta 1979 y desde 1980.

Todos los análisis implementados indican claramente la existencia de un efecto Cuaresma que se va debilitando (y contrayendo) con el tiempo, y que desaparece definitivamente a partir de la década de los ochenta (ver Figura 3 y Tabla 2). Estos análisis coinciden con los resultados encontrados en un estudio sobre la población ortodoxa rumana (Herteliu et al. 2015), y con los encontrados por Reher (1988) para la población de Cuenca de los siglos XVIII y $\mathrm{XIX}$, donde las concepciones durante la Cuaresma (controladas para la fecha exacta de la Pascua) se reducían más del 20 por ciento en relación con lo que deberían haber sido sin ninguna estacionalidad. A modo de ejemplo, la Figura 3 muestra los $p$-valores obtenidos para el efecto Cuaresma al ajustar la serie de concepciones del conjunto de Andalucía para distintos periodos: 1901-1979 (panel superior izquierdo), 1921-1979 (panel superior derecho), 1941-1979 (panel inferior izquierdo) y 1980-2002 (panel inferior derecho). Como se observa claramente, el efecto Cuaresma es estadísticamente significativo (en un nivel de significación del 5\%) desde 40 días antes del Domingo de Resurrección, cuando consideramos la serie desde 1901. A medida que vamos acortando la longitud de la serie, el efecto se va retrasando en el tiempo. En concreto, cuando la serie arranca

Figura 2.

Distribución mensual de concepciones en Andalucía a lo largo del siglo XX. Panel izquierdo: Porcentaje mensual de concepciones por décadas (coeficiente clásico de estacionalidad). Panel derecho: Serie mensual de fechas de concepciones para las personas nacidas en Andalucía y con vida a 1 de enero de 2003.
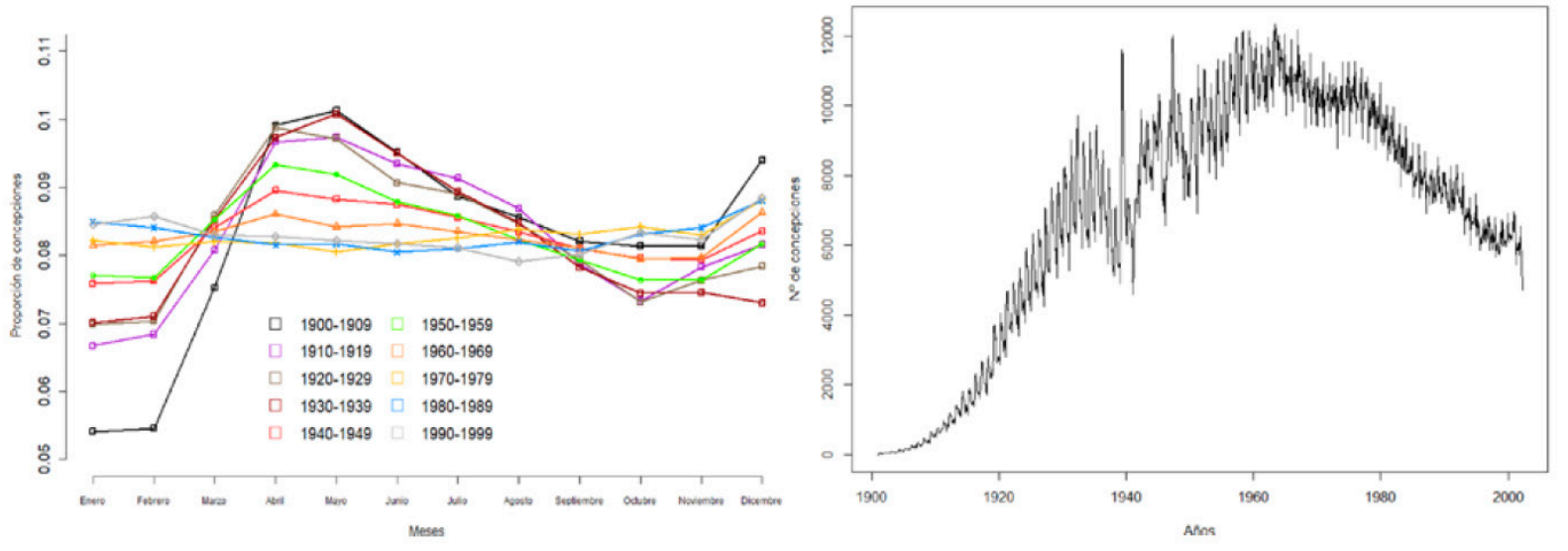

RIS [online] 2018, 76 (3), e107. REVISTA INTERNACIONAL DE SOCIOLOGÍA. ISSN-L: 0034-9712 
en 1921 o en 1941 el efecto es significativo desde 33 días antes. A partir de 1980 el efecto Cuaresma desaparece. El coeficiente asociado no es estadísticamente significativo (tampoco al 10\%) para ninguna longitud del efecto Cuaresma desde 1980.

Finalmente, a fin de reforzar las evidencias anteriores y mostrar otros aspectos, también interesantes, que se derivan del ajuste mediante modelos regARIMA de las series de concepciones, la Tabla 2 presenta los ajustes obtenidos para las series de concepciones del conjunto de Andalucía correspondientes a los periodos de 1930 a 1979, por una parte; y de 1980 a 2002, por otra, incluyendo un efecto Cuaresma que comienza 30 días antes del Domingo de Resurrección.

Como se observa en la Tabla 2 y en la Figura 3, el efecto Cuaresma es estadísticamente significativo en el periodo 1930-1979 y deja de serlo en el periodo
1980-2002. Respecto a los otros efectos de calendario, se observa que: (i) el efecto año bisiesto es significativo en ambas series (es decir, que en los años en que febrero tiene 29 días se producen más concepciones que los años en que tiene 28 días); y (ii) el efecto ciclo semanal no es estadísticamente significativo en el periodo 1930-1979 y sí lo es a partir de 1980. Es decir, que a partir de 1980 se producen más concepciones en los meses con más fines de semana. ${ }^{5}$

Además de las conclusiones anteriores, se observa otro hecho sobresaliente en la serie de concepciones de 1930 a 1979. Se detectan cuatro outliers o valores atípicos. Coincidiendo con el final de la Guerra Civil española (abril de 1939), se produce un incremento puntual (outlier aditivo: OA1939.Abr) de las concepciones que provoca un cambio de nivel que se mantiene hasta agosto de ese mismo año (cambios de

Figura 3.

P-valores para el regresor del efecto Cuaresma, en función del número de días, para la serie de concepciones del conjunto de Andalucía. Panel superior izquierdo: 1901-1979. Panel superior derecho: 1921-1979. Panel inferior izquierdo: 1941-1979. Panel inferior derecho: 1980-2002.
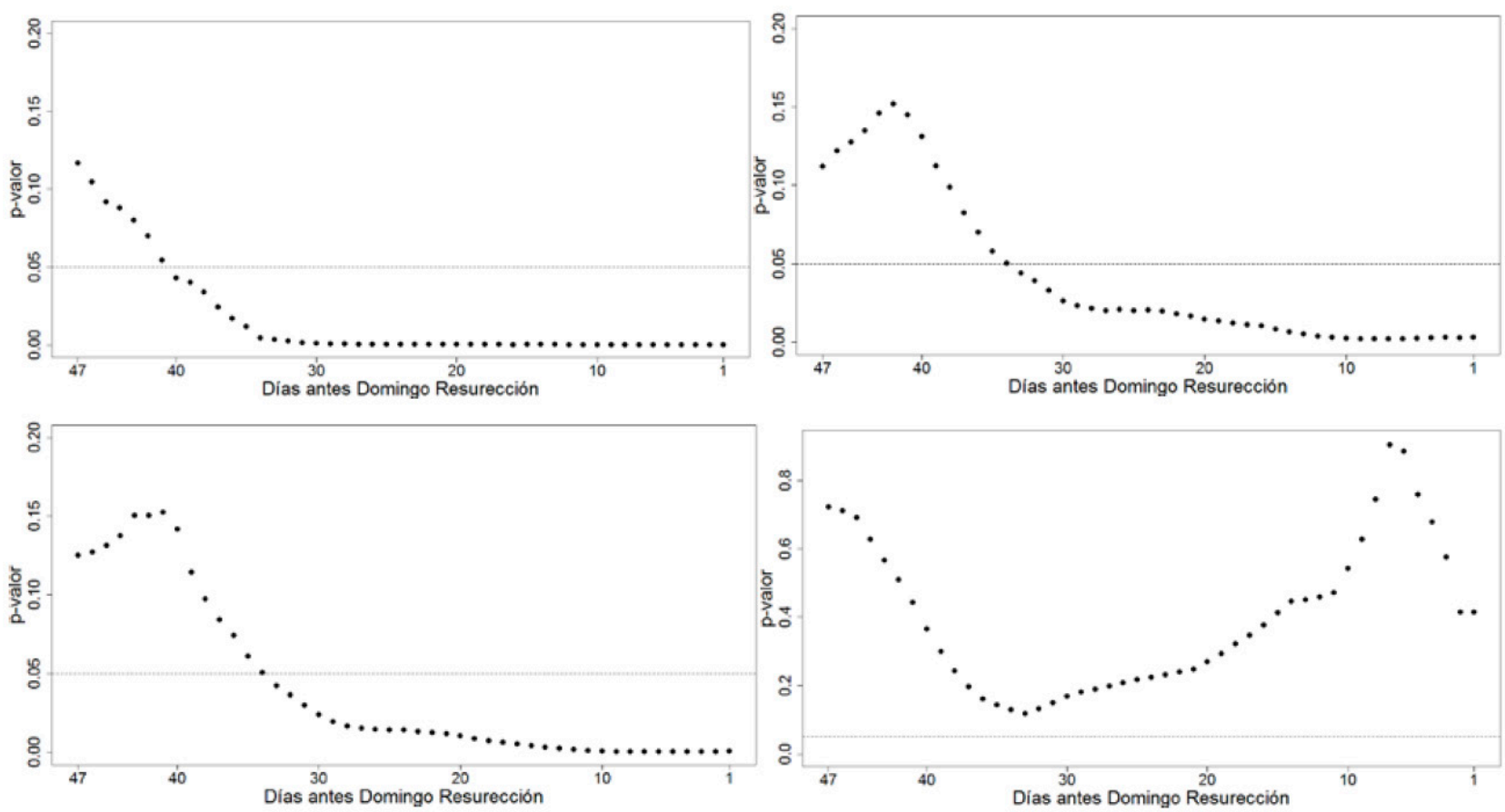

Tabla 2.

Ejemplos de ajustes de modelos regARIMA para las concepciones en Andalucía.

\begin{tabular}{|lllll|}
\hline Regresor & \multicolumn{1}{c}{ Concepciones: 1930-1979 } & \multicolumn{2}{c|}{ Concepciones: 1980-2002 } \\
\hline Efecto Cuaresma (30 días) & Significativo & p-valor: 0.02344 & No significativo & p-valor: 0.16800 \\
Efecto ciclo semanal & No significativo & p-valor: 0.99127 & Significativo & -valor: 0.00008 \\
Efecto año bisiesto & Significativo & p-valor: 0.00299 & Significativo & p-valor: 0.05010 \\
Componente estacional MA(12) & Significativo & p-valor: 0.00000 & Significativo & p-valor: 0.00000 \\
Componente no estacional MA(1) & No identificado & - & Significativo & -valor: 0.00000 \\
Valores atípicos detectados & OA1939.Abr, CN1939.Abr, CN1939.Ago, & & - \\
& CN1957.Dic & & & - \\
\hline
\end{tabular}


nivel CN1939.Abr y CN1939.Ago), momento a partir del cual la serie vuelve a su senda habitual. El análisis estadístico revela cómo, coincidiendo con el reencuentro de las parejas que habían estado separadas durante la contienda, aumentaron las concepciones con el final de la Guerra Civil. Finalmente, en diciembre de 1957 se detecta otro atípico cambio de nivel, cuya interpretación escapa a nuestro conocimiento.

\section{Efecto rebote y erosión del precepto religioso}

En poblaciones pre-modernas sin acceso a los métodos anticonceptivos y de planificación familiar es esperable que, tras periodos en los que disminuye la probabilidad de mantener relaciones sexuales, se produzca un efecto de repunte de las concepciones (Reher 1988; Torrents 1995; Masdéu 2015, entre otros).

En esa línea, nuestros resultados corroboran la existencia de este efecto rebote. La Figura 4 (panel superior derecho), muestra el resultado del ratio 2 . En la primera parte del siglo XX, tenían lugar aproximadamente un $20 \%$ más de concepciones durante el periodo posterior a la Cuaresma que durante dicho periodo. Este porcentaje disminuye paulatinamente hasta situarse en torno al $5 \%$ en $1950 \mathrm{y}$, posteriormente, se observa una erosión hasta su completa desaparición en los últimos 25 años del periodo de estudio. Este primer ratio utilizado pone en evidencia un menor número de concepciones durante el conjunto del periodo de Cuaresma. Las mismas conclusiones, aunque amplificadas, se infieren observando el ratio 3 (Figura 4, panel inferior izquierda), que elimina el efecto de los partos prematuros, comparando además el periodo núcleo de la Cuaresma con un periodo de la misma longitud posterior.

En los resultados de los ratios 2 y 3 , sin embargo, puede interferir la estacionalidad de los nacimientos, consecuencia del impacto de las condiciones climatológicas y de fotoperiodo que han señalado estudios precedentes (Cancho-Candela, Andrés-de Llano y Ardura-Fernández 2007; Quesada 2006). Los resultados encontrados con el cálculo del ratio 1 (Figura 4, panel superior izquierdo), correspondientes a la comparación del número de concepciones durante la Cuaresma y del mismo número de días del periodo inmediatamente anterior, evidencian que, como se esperaba, durante el periodo de invierno el número de concepciones fue, en la primera mitad

\section{Figura 4}

Ratios de concepciones en el conjunto de Andalucía. Superior izquierda $\left(r_{1}\right)$ : ratio que compara las concepciones 47 días antes de la Cuaresma y durante el periodo de Cuaresma. Superior derecha $\left(r_{2}\right)$ : ratio que compara las concepciones 47 días después de la Cuaresma y el periodo de Cuaresma. Inferior izquierda $\left(r_{3}\right)$ : ratio que compara las concepciones entre $t+7$ y $t+33$ y durante el periodo central de Cuaresma (t-40 y $t$-14). Inferior derecha $\left(r_{4}\right)$ : ratio que compara las concepciones entre los últimos y primeros 7 días antes y después de la

Cuaresma y el periodo central de la misma (t-21 y t-7). Nótese la diferente escala de cada panel.
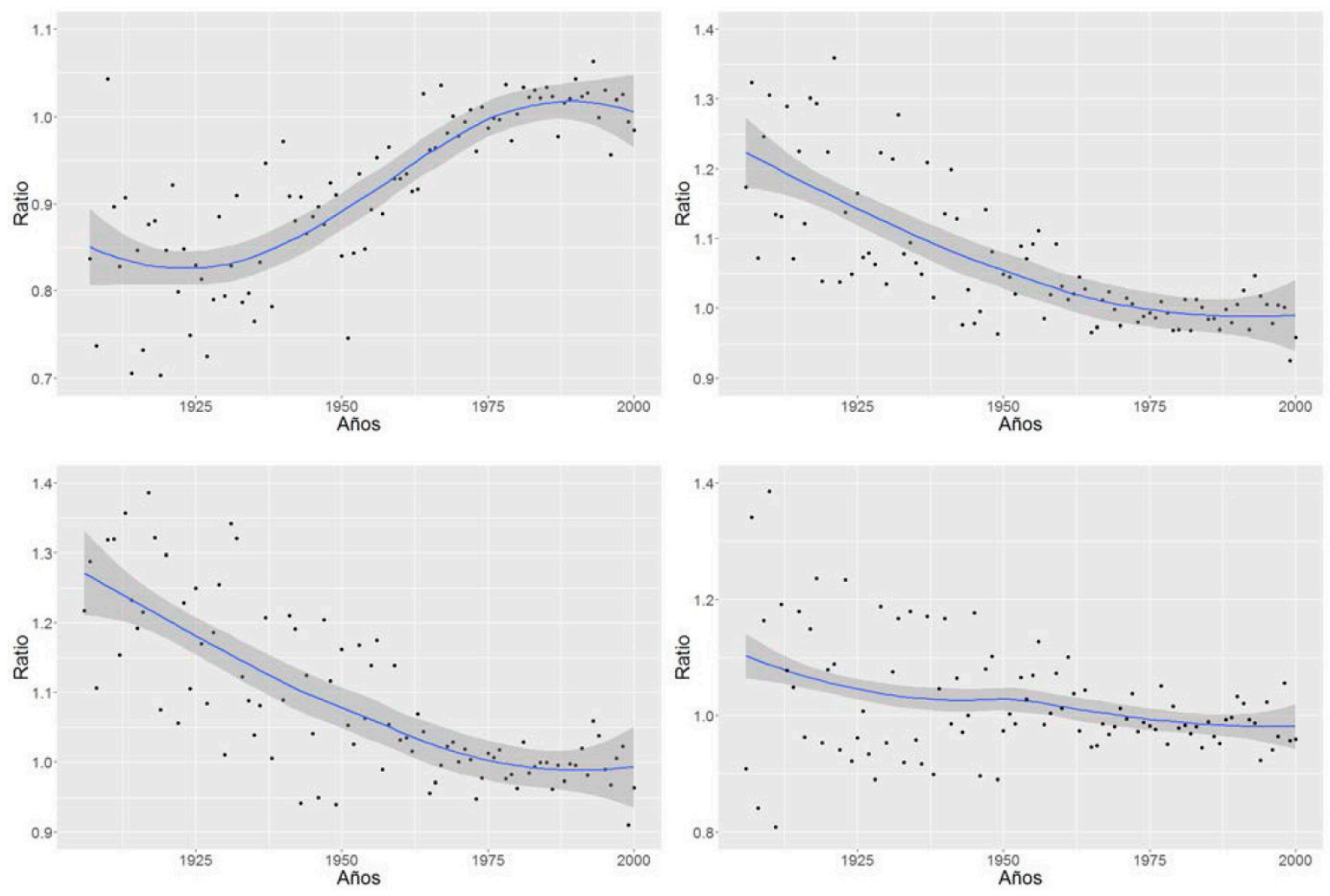
del siglo XX, menor que en el periodo de Cuaresma, coherente con el patrón de la estacionalidad propia de poblaciones pre-modernas. No obstante, también se observa que, a pesar de mantenerse los patrones estacionales ambientales durante gran parte del siglo, los ratios fueron convergiendo de forma progresiva hacia la unidad. Este resultado también es compatible con la hipótesis de una pérdida paulatina de influencia entre la población del precepto religioso.

Para mitigar los problemas relacionados con la interferencia de los efectos de estacionalidad en los ratios 2 a 3 , se calcula el ratio 4 . El panel inferior derecha de la Figura 4 muestra un claro efecto rebote, una vez finalizado el periodo de Cuaresma, para los años que transcurren entre 1900 y 1975 . El efecto desaparece para el resto de los años. El valor anclado en la unidad que muestra la curva ajustada a partir del 1975 aporta evidencia de que, a partir de esa fecha, desaparece el efecto rebote y da idea de la práctica desaparición del efecto Cuaresma y de la interdicción sexual que la acompañaba.

\section{Proceso de difusión de ámbitos municipales de gran tamaño a ámbitos más pequeños}

La incorporación de la mujer al mercado de trabajo, así como la ampliación y extensión del derecho a la educación han provocado grandes cambios en las pautas reproductivas y en las estructuras familiares españolas durante el siglo XX (Del Campo Urbano 1982; Iglesias de Ussel y Flaquer 1993). Las grandes migraciones del campo a la ciudad que se produjeron a partir de la segunda mitad del siglo XX, motivadas principalmente por cuestiones laborales (Capel Sáez 1967), fueron otro factor de transformación igualmente importante. Todo ello ha contribuido a reducir las distancias (no físicas) entre los ámbitos de pequeño y gran tamaño; si bien todavía persisten entre ambos mundos grandes diferencias en multitud de aspectos (p.ej., Camarero, Gómez y Jiménez 1999; Gracia, Gil y Angulo 1998). De hecho, de acuerdo con Fischer (1978), la convergencia es imposible, pues constantemente se están produciendo procesos de difusión hacia el ámbito rural de las innovaciones que se generan en los centros metropolitanos.

Algunos estudios han podido arrojar datos relevantes sobre los procesos de difusión de los cambios en las pautas reproductivas, al analizar la transición de la fecundidad en diferentes territorios (Coale y Watkins 1986). Así pues, es relevante averiguar con que desfase e intensidad se produjo la difusión desde el hábitat de gran tamaño al hábitat de pequeño tamaño, en cuanto al abandono del precepto religioso que prohibía mantener relaciones sexuales durante la Cuaresma, entendido aquel como un rasgo del proceso de secularización que se manifestó primero en las zonas de gran tamaño poblacional y, más tarde, se extendió a las zonas de pequeño tamaño. Y ello a pesar de que, según apuntan Linz y Cazorla (1968), en Andalucía, las poblaciones rurales pudieron ser considerablemente menos religiosas que las urbanas.

Con el objeto de evidenciar este cambio realizamos, con los ratios utilizados en el punto anterior, una comparación entre el hábitat de pequeño tamaño (paneles izquierdos de las figuras $5,6,7$ y 8 ) y el hábitat de gran tamaño (paneles derechos de las figuras $5,6,7$ y 8 ). Como primera evidencia de las comparaciones, se observa en las figuras 6 y 7 los mayores niveles que presentan, al inicio del periodo de análisis, los ratios correspondientes a hábitats de pequeño tamaño, lo que es indicativo de un mayor efecto del precepto religioso en estas zonas. Esto unido al claro desfase que, en su convergencia a la unidad, presentan los ratios en hábitats de pequeño tamaño, revela que el proceso de erosión del precepto religioso que prohibía las relaciones sexuales durante la Cuaresma se manifestó antes y con mayor

Figura 5.

Ratio resultante de comparar el número de concepciones acontecidas entre el periodo comprendido entre t-94 y t-47 días y el periodo de Cuaresma, denotado como el periodo entre $t-47$ y $t$. Hábitats de pequeño tamaño en el panel izquierdo; hábitats de gran tamaño en el panel derecho.
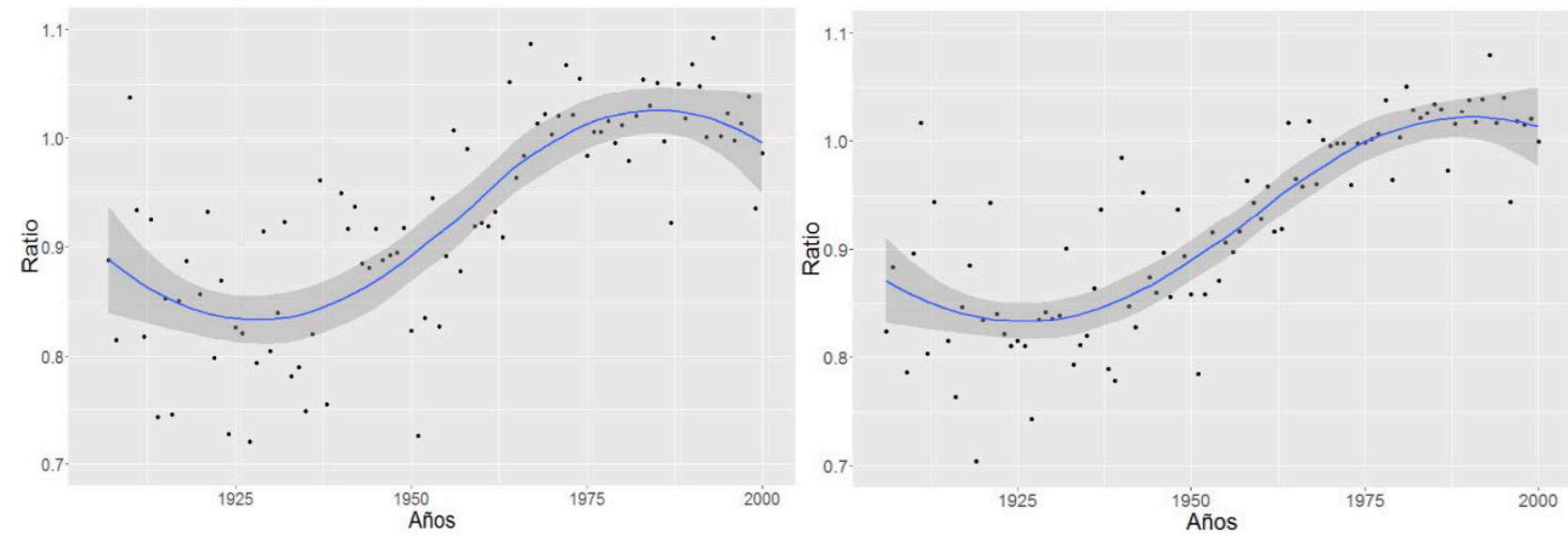
Figura 6.

Ratio resultante de comparar el número de concepciones acontecidas entre el periodo comprendido entre $t$ y t+47 días y el periodo de Cuaresma, denotado como el periodo entre $t-47$ y $t$. Hábitats de pequeño tamaño en el panel izauierdo: hábitats de aran tamaño en el panel derecho.

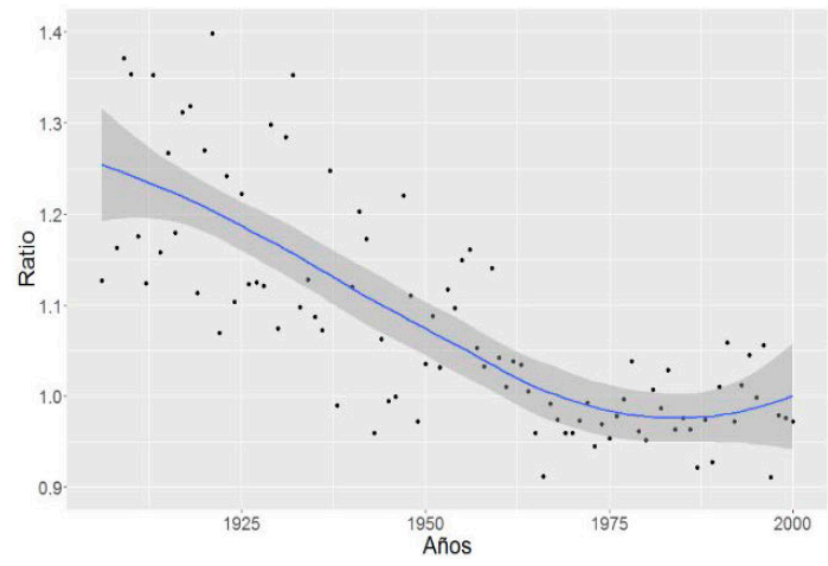

intensidad en los municipios de más de 50.000 habitantes que en los municipios menores de 10.000. En los municipios más grandes el efecto del precepto religioso tiende a ir desapareciendo entre los años 50 y 60 , mientras en los más pequeños este precepto es observable hasta los años 70 .

En la comparación de los ratios de la Figura 5 es más complicado observar diferencias. De hecho, son prácticamente iguales, aunque, como se esperaba, la curva correspondiente a los municipios grandes se sitúa, para el periodo de 1920 a 1970, por debajo de la curva correspondiente a los municipios pequeños. Una posible explicación para esta equivalencia podría ser que, en las ciudades grandes, el impacto sobre las concepciones de los efectos estacionales y de la Cuaresma estarían actuando (relativamente a los municipios más pequeños) en la misma dirección $y$, por tanto, en un ratio se compensarían. Por

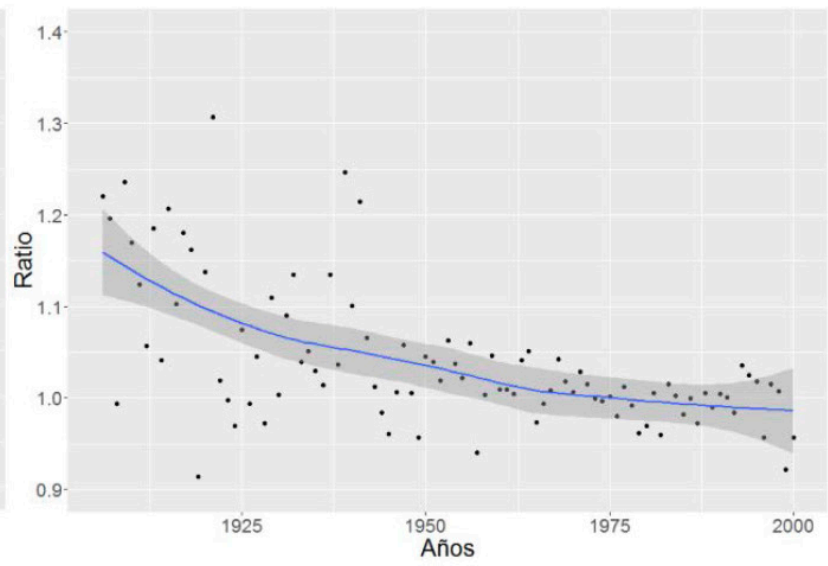

un lado, en las ciudades grandes los inviernos serían menos duros que en los municipios pequeños, por lo que el descenso relativo del número de concepciones sería menor en las grandes ciudades. Por otro lado, la Cuaresma tendría, debido al proceso más rápido de secularización de las ciudades grandes, un menor predicamento en estas zonas, que provocaría también un menor descenso relativo del número de concepciones. Estos dos efectos se compensarían, no mostrando los ratios diferencias significativas. En el caso de los ratios 2 y 3 , este efecto compensador no se daría, pues el efecto incentivador de las concepciones asociado a la primavera de las sociedades pre-modernas actuaría por igual en ambos ámbitos municipales, mientras que el efecto inhibidor de la Cuaresma sería (como ya se ha explicado) superior en los municipios menores de 10.000 habitantes. El análisis de las concepciones mensuales en

Figura 7.

Ratio resultante de comparar el número de concepciones en el periodo comprendido entre $t+7$ y $t+33$ días y el periodo central en la Cuaresma (t-33 y t-14) Hábitats de pequeño tamaño en el panel izquierdo; hábitats de gran tamaño en el panel derecho.
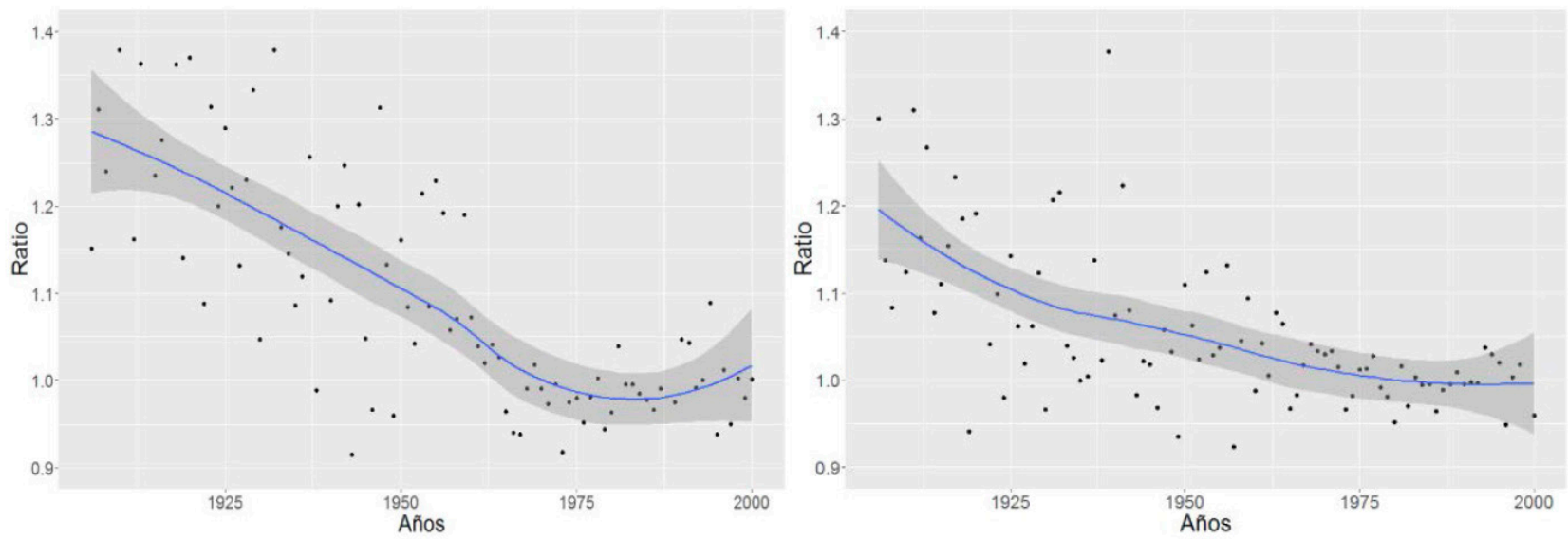
municipios grandes y pequeños, mediante técnicas de series temporales, avala el razonamiento anterior.

Finalmente, el resultado del ratio 4 refuerza las conclusiones anteriores. Eliminando las perturbaciones que los efectos estacionales introducen en los ratios anteriores, el efecto rebote es claramente perceptible en los municipios pequeños, mientras que estadísticamente es inexistente en los municipios más grandes. Este resultado es compatible con la hipótesis de distintos niveles de adherencia a los postulados religiosos, según el tamaño de los municipios.

Los resultados obtenidos en este apartado refuerzan la hipótesis de que se produjo una difusión de los municipios más grandes a los más pequeños, al mostrar que existe una fuerte diferencia en la observación del precepto religioso durante la Cuaresma tanto en la datación de la erosión como en su intensidad.

\section{Conclusiones}

En el contexto de mayor necesidad en la toma de decisiones individualizada que caracterizó la transición de la fecundidad, analizar el papel de la religión en las pautas reproductivas es de interés para cualquier sociedad, también en el caso de Andalucía. El análisis de la estacionalidad de las concepciones de las personas nacidas en Andalucía entre 1901 y 2002 muestra que, durante dicho periodo, se produjo una profunda transformación coincidiendo con el proceso de fuerte reducción de la fecundidad hasta niveles muy inferiores al reemplazo generacional.

Nuestros resultados muestran que la población fértil tendió a respetar el precepto religioso que prohibía las relaciones sexuales durante la Cuaresma, lo que se tradujo en una disminución de concepciones durante dicho periodo y en un aumento de las concepciones tras este, como consecuencia de un efecto rebote. Con ello, queda probado que los factores socioculturales, en este caso como consecuencia de la observación de las directivas morales católicas, fueron muy importantes en Andalucía antes y durante la transición de la fecundidad; periodo que precede a la extensión del uso de los métodos anticonceptivos y la planificación familiar.

Con los resultados que hemos recogido se ofrece evidencia robusta de que la estacionalidad premoderna de los nacimientos no estaba sólo regulada por el calendario agrícola y por el medio ambiente (aunque estos influyeran en gran medida), sino que también estaba condicionada por factores religiosos que ejercieron un papel relevante. Los factores socioculturales actuaron, sin ningún atisbo de duda, en interacción con limitaciones ecológicas propias de sociedades de subsistencia y con factores climatológicos y energéticos, tales como el fotoperiodo y la temperatura, los cuales modificaban la probabilidad de concebir, al alterar tanto la fertilidad como las posibilidades de mantener relaciones sexuales a lo largo de las estaciones. Cabe señalar que, en nuestros resultados, es difícil disociar el aumento de las concepciones tras la Cuaresma (que hemos llamado efecto rebote) del efecto del fotoperiodo, ya que el fin de la Cuaresma coincide con el crecimiento de la luz y el aumento de la temperatura propios del fotoperiodo que regula la reproducción entre los mamíferos.

Esta investigación también arroja luz sobre cómo se desarrolló a lo largo del siglo XX el proceso de erosión mediante el cual la población dejo de observar el precepto religioso de interdicción de las relaciones sexuales durante la Cuaresma. En Andalucía esta erosión se inició sensiblemente antes en las ciudades grandes, de más de 50.000 habitantes, que en los municipios menores de 10.000 habitantes. Y, en conjunto, su efecto despareció completamente en torno a

Figura 8.

Ratio resultante de comparar el número de concepciones entre el periodo comprendido entre t-7 y $t+7$ días y el periodo central en la Cuaresma (t-24 y t-7). Hábitats de pequeño tamaño en el panel izquierdo; hábitats de gran tamaño en el panel derecho.
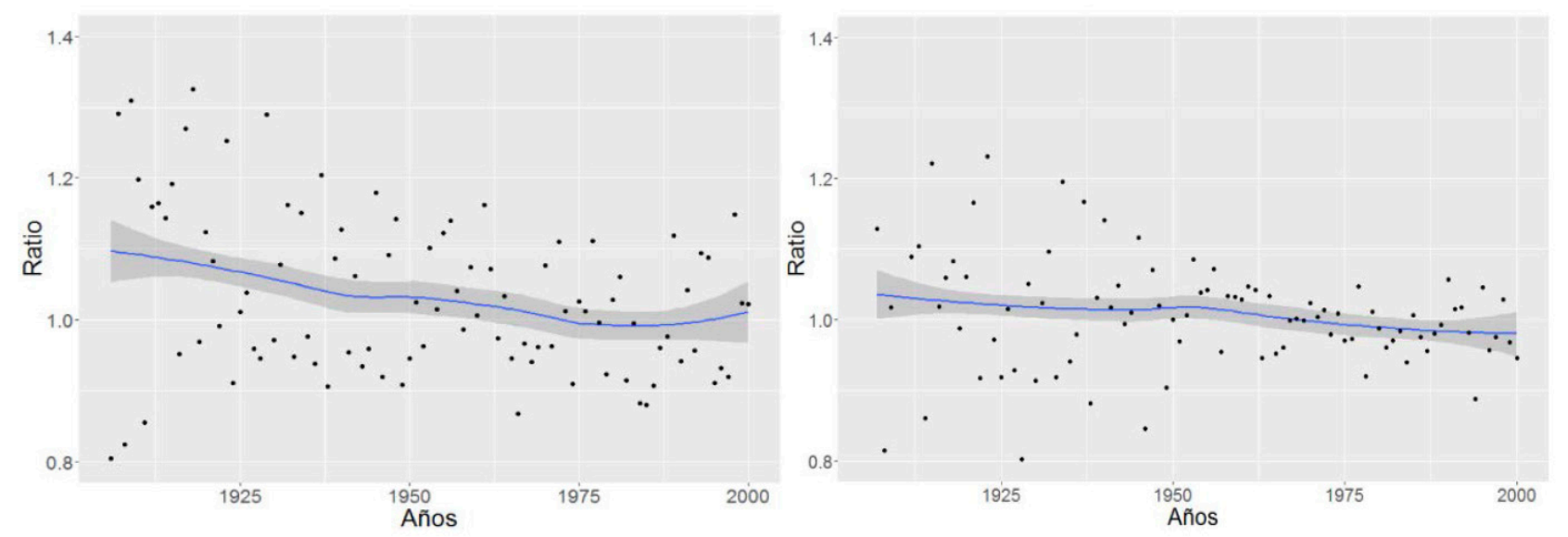
1980, coincidiendo con la última etapa del descenso de la fecundidad, la cual se situó por debajo del umbral de reemplazo y ha permanecido así hasta el día de hoy. A lo largo del siglo XX el indicador coyuntural de fecundidad en Andalucía pasó de 4,47 hijos por mujer en 1915 a 3,2 en 1975 (Instituto de Estadística de Andalucía 1999); y de 2,7 hijos por mujer en 1980 a 1,6 en 1990 y 1,3 en 2000, según el INE.

En nuestra opinión, este proceso es extrapolable a todas las comunidades autónomas españolas, pero queda por probar cuál fue la heterogeneidad regional en torno al papel que tuvo la religión en las pautas reproductivas ( $\mathrm{y}$, más concretamente, en la estacionalidad de las concepciones); cómo se produjo el proceso hacia la modernización y cuáles fueron las pautas temporales. Como en otros aspectos socioculturales, las diferencias regionales en lo concerniente al peso

\section{Notas}

1. Probabilidad de que una mujer que mantiene relaciones sexuales de forma regular sin usar métodos anticonceptivos quede embarazada durante una ovulación.

2. En un primer análisis consideramos como hábitat de gran tamaño los municipios de más de 100.000 habitantes, y como hábitat de pequeño tamaño los municipios con menos de 25.000 habitantes. Esta otra delimitación arroja resultados similares a los obtenidos en el presente trabajo.

3. Wrigley y Schofield (1981) y Wrigley et al. (1997) utilizan una variación del cociente estacional ideado por Louis Henry (1967) en la que dividen dos cocientes: el número

\section{RefERENCIAS BibliogRÁficas}

Adserà, A. 2006. "Marital fertility and religion in Spain, 1985 and 1999". Population Studies 60: 205-221. https://doi. org/10.1080/00324720600684817

Bazán, I. 2008. "El modelo de sexualidad de la sociedad cristiana medieval, norma y transgresión". Cuadernos de CEMyR 16: 167-191.

Bentley, G.R. A.M. Harrigan, B. Campbell y P.T. Ellison. 1993. "Seasonal effects on salivary testosterone levels among Lese males of the Ituri Forest, Zaire". American Journal of Human Biology 5: 711-717. https://doi. org/10.1002/ajhb.1310050614

Bobak, M. y A. Gjonca. 2001. "The seasonality of live birth is strongly influenced by socio-demographic factors". Human Reproduction 16: 1512-1517. https://doi. org/10.1093/humrep/16.7.1512

Box, G.E. y G.M. Jenkins. 1976. Time Series Analysis: Forecasting and Control (Second Edition). San Francisco, CA: Holden-Day.

Breschi, M. y M. Livi-Bacci. 1994. "Le mois de naissance comme facteur de survie des enfants". Annales de Démographie Historique 1: 169-186.

Cabanes, P. 2003. "La sexualidad en la Europa medieval cristiana”. LEMIR. Revista Electrónica sobre Literatura Española Medieval y Renacimiento 7. Departamento de Filología Española. Facultad de Filología. Universitat de València. http://parnaseo.uv.es/Lemir/Revista/Revista7/ Revista7.htm [consultado el 18/05/2008]. de la religión en las pautas reproductivas han de ser el objeto de un análisis pormenorizado. Ello es substancial en la comprensión del proceso de modernización en general y de la transición a la fecundidad más concretamente.

\section{AgradeCIMIENTOS}

Los autores agradecen a los participantes en el VIII Congreso Andaluz de Sociología y a dos evaluadores anónimos los comentarios realizados, y a Christoph Sax su ayuda con la librería seasonal. Cualquier inexactitud que exista es, por supuesto, responsabilidad única de los autores. Los autores reconocen, asimismo, el apoyo recibido por los proyectos CSO2013-43054-R ECO2017-87245-R del Ministerio de Economía, Industria y Competitividad.

de nacimientos del mes dividido por el número de días del mes; y todo ello ponderado por la división entre los nacimientos totales del año y 365,3 .

4. Léridon (1973) avanzó en el desarrollo de estas técnicas para el estudio de la estacionalidad de los nacimientos.

5. Probablemente, este último efecto vendría motivado por causas de organización social, dado que en los últimos años hemos asistido a una concentración de los tiempos de ocio (y de trabajo) en las mismas semanas del año y los mismos días de la semana (Prieto et al. 2008).

Camarero, L.A., R. Gómez y R. Jiménez. 1999. "Morir en el campo y en la ciudad: análisis de las diferencias urbano-rurales en mortalidad". Revista Internacional de Sociología 23: 97-129.

Cancho-Candela, R., J.M. Andrés-de Llano y J. ArduraFernández. 2007. "Decline and loss of birth seasonality in Spain: analysis of 33421731 births over 60 years". Journal of Epidemiology \& Community Health 61: 713-18. https://doi.org/10.1136/jech.2006.050211

Capel Sáez, H. 1967. "Los estudios acerca de las migraciones interiores en España". Revista de Geografía 1: 77-101.

Christoph, S. 2016. Seasonal. $R$ Interface to X-13ARIMASEATS. $R$ package version 1.3.0. Swiss State Secretariat of Economic Affairs. https://CRAN.Rproject.org/package=seasonal [consultado el 18/05/2008].

Cleveland, W.S., E. Grosse y W.M. Shyu. 1992. "Local regression models". Pp. 309-376 en Statistical Models in S, editado por J.M. Chambers y T.J. Hastie. Wadsworth $\&$ Brooks/Cole.

Coale, J. 1973. "The Demographic Transition Reconsidered. International Union for the Scientific Study of Population". En Proceedings of the International Population Conference. Lieja: Éditions Ordina.

Coale, A.J. y S.C. Watkins [eds.] 1986. The Decline of Fertility in Europe: the Revised Proceedings of a Conference 
on the Princeton European Fertility Project. Princeton: Princeton University Press.

Condon, R. y R. Scaglion. 1982. "The ecology of human birth seasonality". Human Ecology 10: 495-510.

Cordero, J. 2009. "El espaciamiento de los nacimientos: una estrategia para conciliar trabajo y familia en España". Revista Española de Investigaciones Sociológicas 128: $11-33$

Del Campo Urbano, S. 1982. La evolución de la familia española en el siglo XX. Madrid: Alianza.

Díaz-Salazar, R. 1993. "La transición religiosa de los españoles". Pp. 93-174 en Religión y sociedad en España, editado por R. Díaz-Salazar y S . Giner. Madrid: Centro de Investigaciones Sociológicas.

Dijkstra, L. y H. Poelman. 2008. "Remote Rural Regions: How the proximity to a city influences the performances of rural regions". Regional Focus 1/2008. Brussels: DGRegio.

Ellison, P., C. Valeggia y D. Sherry. 2005. "Human birth seasonality". Pp. 379-399 en Seasonality in Primates: Studies of Living and Extinct Human and Non-Human Primates. Cambridge University Press.

Fischer, C.S. 1978. "Urban-to-Rural Diffusion of Opinions in Contemporary America". American Journal of Sociology 84: 151-159.

Fuster, V. 1989. "Seasonality of births and family characteristics in a Spanish population". Journal of Biosocial Science 21: 465-474. https://doi.org/10.1017/ s0021932000018204

Gracia, A., J.M. Gil y A.M. Angulo. 1998. "El consumo de alimentos en España: el consumidor rural versus urbano". Estudios Regionales 50: 111-129.

Goerlich, F.J. e I. Cantarino. 2015. "Estimaciones de la población rural y urbana a nivel municipal". Estadística Española 186: 5-28.

Gual i Vilà, V. 1988. Vida i mort a la Conca de Barberà a l'edat moderna (Rocafort de Queralt s. XVI-XVIII). Tarragona: Institut d'Estudis Tarraconenses Ramon Berenguer IV.

Haandrikman, K. 2004. "Seasonality of Births in the Netherlands: Changing Patterns Due to Conscious Planning?". Ponencia presentada en el Annual Meeting of the Population Association of America. Boston, Massachusetts, 1-3 de abril 2004.

Henry, L. 1961. "Some data on natural fertility". Eugenics Quarterly 8: 81-91. https://doi.org/10.1080/19485565. 1961.9987465

Henry, L. 1967. Manuel de Démographie Historique. GenèveParis: Droz-Minard.

Herteliu, C., B.V. lleanu, M. Ausloos y G. Rotundo. 2015. "Effect of religious rules on time of conception in Romania from 1905 to 2001". Human Reproduction 30: 22022214. https://doi.org/10.1093/humrep/dev129

Iglesias de Ussel, J. LI. y Flaquer. 1993. "Familia y análisis sociológico en España". Revista Española de Investigaciones Sociológicas 61: 57-75. https://doi. org/10.2307/40183617

Instituto de Estadística de Andalucía. 1999. Un siglo de demografía en Andalucía: la población desde 1900. Sevilla: Instituto de Estadística de Andalucía. https://www. juntadeandalucia.es/institutodeestadisticaycartografia/ sid/pub/UnSigloDeDemografiaAnd.pdf [consultado el 18/05/2018].
Iriso, P.L. y D.S. Reher. 1987. "La fecundidad y sus determinantes en España, 1887-1920. Un ensayo de interpretación". Revista Española de Investigaciones Sociológicas 39: 45-118. https://doi. org/10.2307/40183293

Knodel, J. 1988. Demographic Behavior in the Past: A Study of Fourteen German Village Populations in the Eighteen and Nineteenth Centuries. Cambridge: Cambridge University Press.

Knodel, J. y E. Van de Walle. 1979. "Lessons from the Past: Policy Implications of Historical Fertility Studies". Population and Development Review 5: 217-245. https://doi.org/10.2307/1971824

Lam, D. y J. Miron. 1991. "Temperature and the seasonality of births". Advances in Experimental Medicine and Biology 286: 73-88. https://doi.org/10.1007/978-14684-5913-5 7

Lam, D. y J. Miron. 1994. "Global patterns of seasonal variation in reproductive outcomes? The European Study Group on Infertility and human fertility". Annals of the New York Academy of Sciences 709: 9-28. https://doi. org/10.1111/j.1749-6632.1994.tb30385.x

Léridon, H. 1973. Natalité, Saisons et Conjoncture Économique. Paris: PUF.

Lesthaeghe, R. 1980. "On the social control of human reproduction". Population and Development Review 6: 527 548. https://doi.org/10.2307/1972925

Lesthaeghe R.J. y C. Wilson. 1986. "Modes of production, secularization and the pace of the fertility decline in Western Europe, 1870-1930". Pp. 261-292 en The Decline of Fertility in Europe, editado por A. J. Coale and S. C. Watkins. Princeton, New Jersey: Princeton University Press.

Liefbroer, A.C., J. Klobas, D. Philipov e I. Ajzen. 2015. Reproductive Decision-making in a Macro-micro Perspective: A Conceptual Framework. Amsterdam: Springer.

Linz, J.J. y J. Cazorla. 1968. "Religiosidad y estructura social en Andalucía: la práctica religiosa". Anales de Sociología 4-5: 3-24.

Lledó, J., J.M. Pavía y F.G. Morillas. 2017. "Transformations in weekly birth distribution. Temporal analysis 1940-2010". Revista Española de Investigaciones Sociológicas, 159: 151-162. http://dx.doi.org/10.5477/cis/reis.159.151

Masdéu, R. 2015. "Resconstrucció de Famílies de la Parròquia de Sant Just Desvern (1509-1936). Un Estudi de Demografía Histórica". Tesis doctoral. Departament d'Història Moderna i Contemporània. Universitat Autònoma de Barcelona. Barcelona.

OECD. 2012. Redefining "Urban": A New Way to Measure Metropolitan Areas. París: OECD. http://dx.doi. org/10.1787/9789264174108-en

OMS. 2012. Nacidos Demasiado Pronto. Informe de Acción Global sobre Nacimientos Prematuros. Nueva York: March of Dimes, PMNCH, Save the Children, Organización Mundial de la Salud.

Prieto, C., R. Ramos y J. Callejo. 2008. Nuevos Tiempos del Trabajo. Entre la Flexibilidad Competitiva de las Empresas y las Relaciones de Género. Madrid: Centro de Investigaciones Sociológicas.

Quesada, A. 2006. "Cambios en la estacionalidad de los nacimientos en Andalucía, España, entre 1941 y 2000". Boletín de la Real Sociedad Española de Historia Natural (Sec. Biológica) 101: 77-85. 
R Core Team. 2016. R: A Language and Environment for Statistical Computing. Vienna: $R$ Foundation for Statistical Computing. http://www.R-project.org/

Ramírez, F. 1998. "Transformaciones y comportamientos demográficos deferenciales en dos municipios del interior andaluz. Aplicación del método de reconstrucción de familias en las poblaciones de Iznájar y Loja (siglos XVIII-XX)". Tesis Doctoral. Departamento de Geografía Humana. Universidad de Granada. Granada.

Reher, D. 1988. Familia, Población y Sociedad en la Provincia de Cuenca. 1700-1970. Madrid: Centro de Investigaciones Sociológicas.

Reher, D. y A. Sanz Gimeno. 2006. "Marked from the outset: season of birth and health during early life in Spain during the demographic transition". Continuity and Change 21: 107-129. https://doi.org/10.1017/S0268416006005765

Requena, M. 2005. “The secularization of Spanish society: change in religious practice". South European Society \& Politics 10: 369-390. http://dx.doi.org/10.1080/13608740500281902

Ruiz, M. T., A. Cabré, T. Castro y M. Solsona. 2005. Anticoncepción y Salud Reproductiva en España: Crónica de una (R)evolución. Madrid: Consejo Superior de Investigaciones Científicas.

Rusell, D., A. Douglas y T. Allan. 1993. "Changing seasonality of birth-a possible environmental effect". Journal of Epidemiology and Community Health 47: 362-367. https://doi.org/10.1136/jech.47.5.362

Sánchez Barricarte, J.J. 2006. "Reproductive behaviour in three Navarrese villages (eighteenth to twentieth century)".
Continuity and Change: A Journal of Social Structure, Law and Demography in Past Societies 21: 419-454.

Torrents, À. 1995. "Transformacions Demogràfiques en un Municipi Industrial Català: Sant Pere de Riudebitlles, 16081935". Tesis Doctoral. Departament d'Història i Institucions Econòmiques. Universitat de Barcelona. Barcelona.

Uriel, E. y A. Peiró. 2000. Introducción al análisis de series temporales. Valencia: Alfa Centauro.

U.S. Census Bureau. 2016. X-13ARIMA-SEATS Reference Manual. Center for Statistical Research and Methodology: Washington, DC.

Wehr, T.A. 1991. "The durations of human melatonin secretion and sleep respond to changes in day length (photoperiod)". Journal of Clinical Endocrinology and Metabolism 73: 1276-80. https://doi.org/10.1210/jcem-73-6-1276

Wehr, T.A., D.E. Moul, G. Barbato, H.A. Giesen, J.A. Seidel, C. Barker y C. Bender. 1993. "Conservation of photoperiod-responsive mechanisms in humans". American Journal of Physiology 265: 846-857.

Wickham, H. 2009. ggplot2: Elegant Graphics for Data Analysis. New York: Springer-Verlag.

Wrigley, E.A., R. Davies, J. Oeppen y R. Schofield. 1997. English Population History from Family Reconstitution 1580-1837. Cambridge: Cambridge University Press.

Wrigley, E.A. y R. Schofield. 1981. The Population History of England, 1541-1871: A Reconstruction. Cambridge: Cambridge University Press.

JOSEP LLEDÓ es diplomado en Ciencias Empresariales, licenciado en Ciencias Actuariales y Financieras, máster en Ciencias Actuariales y Financieras y doctorando en Estadística y Optimización. Sus áreas de interés incluyen la estadística, la demografía, la sociología y el análisis de riesgos

CARLES X. SIMÓ-NOGUERA es doctor en Demografía por la Université de Montréal (Quebec, Canadá), es profesor titular en la Universitat de València, donde se incorporó mediante el programa Ramón y Cajal para la contratación de doctores. Ubicada principalmente en los campos científicos de la demografía y la sociología, su investigación se ha centrado en sistemas de información poblacional, el divorcio, la demografía de la familia, los ciclos y transiciones vitales, la inmigración y el envejecimiento.

JOSE M. PAVÍA es licenciado en Ciencias Matemáticas y doctor en Ciencias Económicas, es catedrático de Métodos Cuantitativos en la Universitat de València. Pavía desarrolla y aplica métodos empíricos en diversos ámbitos de investigación en ciencias sociales. Sus áreas de interés incluyen la sociología, la estadística espacial, la demografía, el muestreo, la ciencia política, las predicciones electorales y la inferencia ecológica. 